\title{
MedienPädagogik
}

Zeitschrift für Theorie und Praxis der Medienbildung

\section{Die Dualität von Kompetenz in tiefgreifend mediatisierten Welten}

\section{Zum Verständnis eines theoretischen Schlüsselkonzepts der Medienpädagogik}

\author{
Henriette Mehlan und Jörg Holten
}

\section{Zusammenfassung}

In tiefgreifend mediatisierten Welten ist Medienkompetenz ein Schlüsselbegriff. Für Politik, Wirtschaft und Bildung ebenso wie für Wissenschaft. Das Konzept Medienkompetenz und vor allem sein Gehalt für die Wissenschaft sind aber umstritten. Innerhalb des medienpädagogischen Diskurses hat es unterschiedliche Wendungen erfahren, die zuletzt dazu führten, dass es ganz abgelehnt wurde. Der Beitrag zeichnet die definitorischen Wendungen nach und legt schliesslich ein eigenes Verständnis vor. Dabei werden Kompetenz und Performanz im Anschluss an Giddens als zwei Dimensionen unterschieden (Dualität), die sich gegenseitig bedingen und reproduzieren. Ferner wird eine zweite Unterscheidung eingeführt, die zwischen Wirklichkeitskonstruktionen erster und zweiter Ordnung differenziert. Durch diese Unterscheidungen ist es möglich, bisher implizite Denkweisen über Kompetenz sichtbar zu machen, ohne dabei einer Seite der Dualität von Kompetenz ihre Berechtigung abzusprechen. Aus der vorgeschlagenen Kompetenzdefinition folgt schliesslich, dass das Nachdenken über Medienkompetenz nicht ausschliesslich Aufschlüsselung von einzelnen, vor allem medienbezogenen Kompetenzen sein kann, sondern vielmehr die Frage nach der Dualität von Kompetenz in tiefgreifend mediatisierten Welten impliziert. 


\title{
The duality of competence in deep mediatized worlds
}

\begin{abstract}
In deep mediatized worlds, media competence is a key concept. For politics, economy and education as well as for science. But the concept of media competence and especially its scientific substance are however controversial. Within the media pedagogic discourse, it has undergone different turns and was recently rejected. The article reproduces these turns and finally provides an understanding that takes into account the duality (Giddens) of competence and performance. Competence and performance are distinguished as two dimensions that mutually define and reproduce each other. Further, a second distinction is introduced which differentiates between first and second order constructions of reality. This allows to demonstrate the hitherto implicit meanings of competence without rejecting one side. The proposed definition implicates that thinking about media competence is not primarily a breakdown of individual mediarelated competences, but rather implies the question of the duality of competence in deep mediatized worlds.
\end{abstract}

\section{Einleitung}

Chimäre (Weiner 2011), umbrella term (Wirth 2002) oder Schlüsselkonzept (Süss et al. 2018)? In der Erziehungswissenschaft wurde der (Medien) Kompetenzbegriff immer wieder (kritisch) diskutiert, im medienpädagogischen Diskurs zuletzt sogar beiseitegeschoben (vgl. zur Kritik z. B. in essayistischer Form Weiner 2011; Jarren und Wassmer 2009; Hugger 2008; zum Vorschlag, das Konzept zu ersetzen vor allem Spanhel 2002; 2011; Kerres 2017). Gleichwohl wird er mit Blick auf aktuelle Fragen zur Digitalisierung in unterschiedlichen Kontexten (Politik, Wirtschaft, Bildung, aber auch Wissenschaft) wiederbelebt und als Zielkategorie wiederentdeckt (vgl. Theunert 2011; Theunert und Schorb 2010; Knaus 2018). Darin zeigt sich eines der Hauptprobleme des (Medien)Kompetenzkonzepts, das Vonken (2005, 131) auf den Punkt bringt: 
«Kompetenzen (werden) nicht aus [...] theoretischen Betrachtungen abgeleitet, sondern wirtschafts- und bildungspolitische bzw. unternehmens-strategische Aspekte (spielen) bei ihrer Konstruktion eine Rolle».

Als gesellschaftliche Anforderungen finden diese Aspekte unhinterfragt Eingang in die wissenschaftliche Theoriebildung (vgl. Waldmann und Aktas 2017, 101). Aus dieser «Diversifizierung der Kompetenzinhalte» (Gapski 2001, 196) resultiert eine begriffliche Unschärfe (vgl. Sutter 2014, 73) und normative Überladung des Konzepts im wissenschaftlichen Kontext. Der Medienpädagogik erwachsen daraus Probleme, «theoretisch begründete und empirisch prüfbare Kompetenzmodelle zu entwickeln» (Arnold und Lindner-Müller 2012, 230; vgl. auch Vonken 2005, 11). Die Probleme manifestieren sich innerhalb des (medien-)pädagogischen Diskurses in Form von unterschiedlichen Kompetenzbegriffen, die auf unterschiedliche Verwendungskontexte und Beobachtungslogiken zurückzuführen sind. Diese sollen im Beitrag als 1) nominaler, 2) operationaler und 3) normativer Begriff von (Medien)Kompetenz bezeichnet und voneinander abgegrenzt werden (Abschnitt 2). Dabei wird die Ansicht vertreten, dass die theoretische Begründung, empirische Überprüfung und normative Forderung von Kompetenz nicht in einem Begriff aufgegriffen werden können, da sich ein theoretisch ausgearbeiteter Kompetenzbegriff dem direkten empirischen Zugriff und normativen Ansprüchen entzieht. Um dies zu begründen, wird die Dualität von Kompetenz im Anschluss an Giddens als wechselseitige Beziehung von Kompetenz und Performanz betont (Abschnitt 3.1) und sozialkonstruktivistisch argumentiert (Schütz), dass die unterschiedlichen wissenschaftlichen und gesellschaftlichen Anforderungen gleichermassen Wirklichkeitskonstruktionen zweiter Ordnung sind (Abschnitt 3.2). Sie unterscheiden sich daher grundsätzlich von der Erlebensperspektive von Handelnden.

Für einen medienpädagogischen Medienkompetenzbegriff bedeutet das, dass Medien nicht Bezugsobjekte eines speziellen Kompetenzbereichs sind, sondern Mittel der Wirklichkeitskonstruktion und -bewältigung in tiefgreifend mediatisierten Welten darstellen, wie in Abschnitt 4 gezeigt werden soll. Aufgabe einer Medienpädagogik, so das Fazit in Abschnitt 5, 
wäre es dann nicht, sich mit der Reifizierung von Kompetenz- oder Performanzmodellen zu befassen. Stattdessen gilt es, Fragen zur Medialität von Wirklichkeitskonstruktionen durch Handelnde und den diesen Konstruktionen zugrundeliegenden Bedingungen tiefgreifend mediatisierter Welten zu klären (wie es z. B. von Meder 2011; 2014 angefangen wurde).

\section{Drei Kompetenzbegriffe in der Medienpädagogik}

Zunächst also sollen die unterschiedlichen Kontexte, aus denen heraus der Kompetenzbegriff beansprucht wird, nachgezeichnet werden. Innerhalb des medienpädagogischen Diskurses lassen sich drei implizite Denkweisen identifizieren, die 1) zu einem nominalen, 2) zu einem operationalen und 3) zu einem normativen Begriff von (Medien)Kompetenz geführt haben.

1. Zunächst hat die deutschsprachige Medienpädagogik ihren Kompetenzbegriff von Chomsky, Habermas und Baacke (1980) übernommen. Die Autoren haben vor dem Hintergrund des wissenschaftlichen Anspruchs der Theoriebildung Nominaldefinitionen vorgelegt. Bei Chomsky hat der Kompetenzbegriff den Status einer abstrakten Hypothese über die mentale Realität von Handelnden und meint ein System generativer Prozesse (vgl. Chomsky 1970, 14). Kompetenz ist dabei ein individuelles Konstrukt analytischer Natur, eine «Erfindung von Wissenschaftlern» (Meyer 2010, 147). Von Habermas wurde dieser Ansatz vorbereitend für seine intersubjektivistische Theorie des kommunikativen Handelns als kommunikative Kompetenz weiterentwickelt und Baacke hat, von Habermas ausgehend, den Kompetenzbegriff für die Medienpädagogik als Zielkategorie erstmalig nutzbar gemacht. Durch seine Dimensionierung wurde das Konzept für eine zunehmend handlungsorientierte Medienpädagogik auch praktisch anwendbar. (vgl. dazu ausführlich Tulodziecki 2011, 20-22). Diese Bedeutungsdimension von Kompetenz wird in Abschnitt 3.1. noch einmal aufgegriffen und zu der für diesen Beitrag zentralen Unterscheidung von Kompetenz und Performanz weiterentwickelt. 
2. In einer zweiten Verwendungsweise, die hier als operational bezeichnet wird, ist der Kompetenzbegriff vor allem in der empirischen Bildungswissenschaft eng mit psychometrischen Messmodellen verknüpft. Vor allem Weinert hat mit seiner Definition von Kompetenzen ${ }^{1}$ zum (bildungspolitischen) Wunsch beigetragen, Kompetenz(en) messen und durch Bildungsprozesse fördern zu können. Mit diesem umfangreichen Verständnis von Kompetenzen geht eine Reifizierung des zuvor abstrakten Konstrukts einher, da nun manifeste Indikatoren benannt werden müssen, die erlernt aber auch modifiziert werden können (vgl. Funke 2014, 138). Bereits mit der ersten PISA-Studie, die sich auf die Kompetenzdefinition von Weinert stützt (Baumert et al. 2001, 22), war man sich aber bewusst, dass sich die «Erfassung solcher Handlungskompetenzen» schwierig gestaltet und sich «in der Regel [wird] auf Teilaspekte konzentrieren müssen» (Baumert et al. 2001, 22). In diesem Moment richtet sich der Blick eigentlich von der Kompetenz- auf die Performanzseite, d. h. von der unsichtbaren Tiefenstruktur auf die sicht- und messbare Oberflächenstruktur von Handlung (vgl. Meyer 2010, 147; Abb. 6.5.). Statt dies zu thematisieren und begrifflich zu fassen, wird in Messmodellen aber noch immer von Kompetenz gesprochen. In diesem Sinne wurde und wird der Kompetenz-Begriff dann auch im Plural als «summarische Zuschreibung von Persönlichkeitseigenschaften und Fähigkeiten» (Vonken 2005, 189) gebraucht.

Hieraus ergeben sich allerdings weitreichende empirische Probleme, denn insbesondere

«für die 〈psychosozialen〉 Anteile der Kompetenz - bei Weinert der motivationale, volitionale und z. T. [...] auch der soziale Bereich - [ergeben sich] ernsthafte Probleme einer objektivierbaren Modellierung und damit der Messbarkeit zumindest dieser Anteile» (Rödler 2011, 299),

1 Schon bei ihm wird der Pluralbegriff verwandt. Kompetenzen sind «die bei Individuen verfügbaren oder durch sie erlernbaren kognitiven Fähigkeiten und Fertigkeiten, um bestimmte Probleme zu lösen, sowie die damit verbundenen motivationalen, volitionalen und sozialen Bereitschaften und Fähigkeiten um die Problemlösungen in variablen Situationen erfolgreich und verantwortungsvoll nutzen zu können.» (Weinert 2001, 27f.) 
wie auch Weinert selbst bereits in seinen «Perspektiven der Schulleistungsmessung» $(2001,358)$ anmahnt.

3. Für den schulischen Bereich wurden, trotz dieser Probleme, aus dem operationalen Kompetenzbegriff Bildungsstandards abgeleitet, «hinter deren Erfassung» allerdings «politische Zielsetzungen und Werte stecken» (Funke/Spinath 2014, 144). Eng verbunden mit den Messmodellen ist damit die dritte - als normativ zu bezeichnende - Bedeutung, die den Pluralbegriff weiterführt und Kompetenzen normativ zur Zielkategorie von Bildung und Erziehung erklärt. Diese Bedeutung resultiert gewissermassen aus der Eigenlogik des Erziehungssystems, das von der Annahme ausgeht, es könne und müsse erziehen (vgl. Luhmann 1991; 2002). Wenn Erziehung gemäss dieser Eigenlogik als «planvolle Sozialisation [...] primär darauf ab(zielt), Heranwachsende so zu gestalten, wie die Gesellschaft sie braucht» (Grundmann 2017, 70), meint Kompetenz in pädagogischen Kompetenzmodellen keine Eigenschaft von Individuen, sondern eine normative Zielkategorie (vgl. Süss et al. 2018, 109). In diesem Sinne geht beispielweise Baacke von der Grundannahme aus: «Es ist die 〈Kompetenz〉, die den Menschen einerseits erziehungsbedürftig macht, aber auch erziehungsfähig.» (Baacke 1996, 117) Mit Prämissen wie dieser sind also, in der Eigenlogik des Erziehungssystems gedacht, Medienkompetenzmodelle zwangsläufig normativ. Neben das theoretisch zu fassende Regelsystem, über das Handelnde von sich aus verfügen, tritt, im Zuge einer erziehungswissenschaftlichen Perspektive auf den Begriff Kompetenz, die Bemühung um ein pädagogisch begründetes Regelsystem. Die Chomskysche Ursprungsfrage, wie gelingt es Sprechern (respektive Handelnden) zu sprechen (handeln), wird dann zur normativen: Wie sollten Handelnde handeln?

Normativität, Operationalisierbarkeit und analytische Schärfe - diese drei Ansprüche wurden bisher also an ein und denselben Begriff herangetragen. Sie vermischen sich in gegenwärtigen Medienkompetenzbegriffen, wobei sich von der Chomskyschen Ursprungsbedeutung weit entfernt und stattdessen stärker an den anderen beiden Bedeutungsdimensionen (Messen, Vermitteln) orientiert wird. Dies zeigt sich auch in den Bemühungen 
der KMK (Kultusministerkonferenz), 61 (!) Medienkompetenzen als Bildungsstandards zu formulieren (KMK 2016), die jedoch nicht operationalen Modellen entspringen, sondern normative Vorstellungen repräsentieren und sich einer vergleichenden Leistungsmessung entziehen. Die Vorstellung, Medienkompetenzen als zu vermittelndes instrumentelles Wissen oder als messbare Entwicklungsresultate zu verstehen, wurde in grossen Teilen kritisiert und sich stattdessen an anderen Konzepten (Medienbildung, Mediensozialisation, Media Literacy, etc.) orientiert. Alternative Begriffe lösen das Problem aber nicht. Entsprechend wird im Folgenden versucht, sich auf ursprüngliche Bedeutung von Kompetenz zu besinnen und die anderen beiden Bedeutungsdimensionen davon abzugrenzen.

\section{Kompetenz - Explikation und Systematisierung der impliziten Bedeutungsdimensionen}

Ziel des Abschnitts ist es, die oben beschriebenen Bedeutungen zu ordnen, Kompetenz wieder getrennt von bildungspolitischen und pädagogischen Implikationen zu denken und sich auf die bekannten definitorischen Grundlagen zu besinnen. Dazu werden zwei Ordnungskriterien eingeführt: Zum ersten ist das die Unterscheidung von Kompetenz und Performanz und zum zweiten die Unterscheidung von Wirklichkeitskonstruktionen erster Ordnung und zweiter Ordnung. Es gilt zunächst die Begriffe Kompetenz und Performanz, sowie insbesondere ihr Verhältnis zueinander nominal zu bestimmen. Im Weiteren wird eine eigene Definition von Kompetenz und Performanz vorgelegt und deren Verhältnis zueinander als sich gegenseitig bedingende und reproduzierende Dimensionen derselben Sache verstanden. Diese Beziehung wird, in Anlehnung an Giddens Ausarbeitung des Verhältnisses von Struktur und Handeln, als Dualität von Kompetenz verstanden (Abschnitt 3.1). Mit der anschliessenden Unterscheidung von Wirklichkeitskonstruktionen erster und zweiter Ordnung in Abschnitt 3.2 wird verdeutlicht, dass Kompetenz immer schon ein Zuschreibungsprodukt ist, das von der Erlebensperspektive der Handelnden selbst unterschieden werden muss. 


\subsection{Die Dualität von Kompetenz und Performanz}

Im ersten Schritt soll also die Unterscheidung von Kompetenz und Performanz anhand einiger definitorischer Entwicklungen nachvollzogen und weiterentwickelt werden.

Beide Begriffe stammen aus der (generativen) Grammatiktheorie von Noam Chomsky (1970), waren zunächst also auf Sprache bezogen. In der Linguistik galt es, die Frage zu klären, wie es Sprechern gelingt, zu sprechen. Die Antwort: Sprecher verfügen über eine generative Grammatik (Chomsky 1970, 18), die es ihnen ermöglicht aus einer endlichen Menge von Regeln unendlichen Gebrauch zu machen. Dieses Regelsystem bzw. die Fähigkeit zu seiner Anwendung wurden als Kompetenz bezeichnet und von der Seite der Sprach-Verwendung (Performanz) abgegrenzt. Chomsky $(1970,14)$ betont, in Abgrenzung zu de Saussures Begriff der langue, dass es sich bei Kompetenz um mehr als ein «systematisches Inventar von Einheiten», also um mehr als das Wissen um ein abstraktes Regelsystem handelt. Und zwar um ein «System generativer (〈erzeugender〉) Prozesse» (Chomsky 1970, 14), d. h. um die Fähigkeit der Anwendung des Regelsystems. Ziel des Linguisten war es, von der aktuellen Sprachverwendung (Performanz) auf die nicht beobachtbare Kompetenzseite, die mentale Realität des idealen Sprecher-Hörers, zu schliessen (vgl. Chomsky 1970, 13-14). Denn Beobachtungen der Performanzseite konnten, Chomsky $(1970,14)$ zufolge, kaum Gegenstand einer «ernsthaften Disziplin» sein. Der Schluss von Performanz auf Kompetenz bleibt aber notwendigerweise vage, da sich Kompetenz nicht direkt in Sprachverwendung wiederspiegelt. Sprachverwendung ist eben durch Irregularitäten gekennzeichnet. (vgl. Chomsky 1970, 14)

Dieser linguistische Kompetenzbegriff wurde in der Psychologie in kritischer Auseinandersetzung mit Chomsky weitergedacht und zu einer Handlungskompetenz generalisiert. (vgl. Aebli 1980, 57-58). Aebli (1980) versteht unter Handlungskompetenz

«eine Wissensbasis, die man sich als ein nichtformuliertes Handlungswissen vorstellen muß, und man verbindet damit den Gedanken der Erzeugung von Handlung aus der Wissensbasis heraus, wobei der Schluß naheliegend ist, auch hier anzunehmen, daß aus einer beschränkten Anzahl von Regeln des Handelns die unendlich vielfaltigen 〈Oberflächenstrukturen〉 des Handelns erzeugt werden können.» (Aebli 1980, 58; Hervorheb. i. O.) 
Diese beschränkte Anzahl der Regeln des Handelns beschreibt Aebli (1980, 83 f.) als Handlungsschemata. Handlungskompetenz lässt sich dann als «Repertoire von Handlungsschemata» bestimmen, über das Aktoren verfügen und das sie «in einer aktuellen Situation aktiviere(n)» (Aebli 1980, 99). Handlungsschemata sind wiederholbar und auf neue Situationen übertragbar (vgl. ebd., 84). Aebli unterscheidet also zwischen Wissensbasis als Struktur (Kompetenz), Erzeugung von Handlung (Erzeugung aus der Kompetenz heraus) sowie Oberflächenstrukturen des Handelns (Performanz). Der Aspekt der Erzeugung wird dabei im Gegensatz zu Chomsky als der Wissensstruktur nachgeordnet verstanden, ist also nicht mehr Teil von Kompetenz selbst (vgl. Vonken 2017, 50). Die Oberflächenstrukturen des Handelns lassen sich analog zur Performanz begreifen. Diese Verschiebung war Vonken (2017, 50f.) zufolge Grundstein für die oben beschriebene Entwicklung des Pluralbegriffs von Kompetenz und der «modernen Varianten des Kompetenzbegriffs» (wie sie in Abschnitt 2. beschrieben wurden), in denen die Performanz kaum noch eine Rolle spielt. Will man sich hingegen auf die theoriegeleiteten Hintergründe rückbesinnen, so ist das Verhältnis von Wissensstruktur, Erzeugung und Handeln genauer in den Blick zu nehmen. Zwei Möglichkeiten dazu seien an dieser Stelle angedeutet.

In der sozialphänomenologischen Wissenssoziologie wird das Verhältnis von Wissensstruktur und Erzeugung ebenfalls thematisiert; allerdings wird hier der Fokus eher auf die Seite der Erzeugung von Handeln und Handlungssituationen gelegt. Statt von Wissensbasis im Sinne Aeblis kann sozialphänomenologisch von einem Wissensvorrat gesprochen werden. Dieser subjektive Wissensvorrat baut sich aus grösstenteils sozial bedingten «Sedimentierungen ehemals aktueller, situationsgebundener Erfahrungen» auf (Schütz und Luckmann 2003, 149) und hält diese in Form von Typisierungen als Deutungsschemata für die Auslegung jeweils aktueller Situationen bereit (vgl. ebd., 33). Wissensvorrat und Situationsauslegung sind dabei eng aufeinander bezogen und gewissermassen als gleichzeitig zu denken. Schütz und Luckmann (2003) beschreiben, wie die Auslegung respektive Erzeugung von lebensweltlichen Situationen auf der Basis von verschiedenen Elementen des Wissensvorrats erfolgt. Jede Situation ist den Autoren zufolge einerseits durch die Grundelemente des Wissensvorrats automatisch vorbestimmt (ausgelegt). Sie ist andererseits 
aber zugleich offen, d. h. sie «hat einen unendlichen inneren und äußeren Horizont» (Schütz und Luckmann 2003, 167), der ausgelegt werden muss, damit Handeln überhaupt möglich ist. Auch die Auslegung der offenen Elemente erfolgt allerdings mit Hilfe des Wissensvorrats: zum einen durch das Gewohnheitswissen, zum anderen durch den Wissensvorrat im engeren Sinne. Das Gewohnheitswissen schliesst Fertigkeiten, Gebrauchswissen und Rezeptwissen ein. Während das Gewohnheitswissen ebenso wie die Grundelemente des Wissensvorrats «nicht thematisiert wird, sondern in Situationen und Handlungen automatisch mit einbezogen wird», meint der Wissensvorrat im engeren Sinne ein «System» spezifischer Teilinhalte» (Schütz und Luckmann 2003, 159). Routine-Situationen werden auf Basis des Gewohnheitswissens routinemässig bestimmt, problematische Situation hingegen durch ein bewusstes Besinnen auf den Wissensvorrat im engeren Sinne im Prozess der Horizontauslegung gelöst. (vgl. Schütz und Luckmann 2003, 168f.) Kompetenz lässt sich folglich aus wissenssoziologischer Perspektive als wissensbasierte Fähigkeit zur teils automatisch, teils routinemässig und teils bewusst erzeugten Auslegung von Wirklichkeit definieren. Diese Ausführungen präzisieren Chomskys Hypothese über die mentale Realität der Handelnden als generatives System (Kompetenz). Sie deuten an, wie Wissen und die Erzeugung von Handlungssituationen durch Wissen unmittelbar aufeinander bezogen sind. Kompetenz ist der Erzeugung von Situation und Handlung nie zeitlich vorgelagert, sondern fällt mit der Aktualisierung von Handlungen und der Erzeugung der diese Handlungen kontextualisierenden Situationen zusammen.

Der noch ausstehende Bezug zu den «Oberflächenstrukturen des Handelns» (Aebli) bzw. der Performanz (Chomsky) lässt sich nun aus praxistheoretischer Perspektive herstellen. Mit dem Begriff der sozialen Praktik als Grundeinheit des Sozialen werden Handeln und Wissen programmatisch miteinander verknüpft. «Eine Praktik besteht aus bestimmten routinisierten Bewegungen und Aktivitäten des Körpers. Diese Körperlichkeit des Handelns und der Praktik umfasst die beiden Aspekte der 〈Inkorporiertheit) von Wissen und der 〈Performativität) des Handelns》 (Reckwitz 2003, 290) Insofern schliesst diese Perspektive, zwar in ihrer eigenen Terminologie jedoch sinngemäss an die sozialkonstruktivistischen Grundideen an und versteht Wissen (die generative Wissensbasis) als in der Praxis 
(Performanz) zum Ausdruck kommend (vgl. Reckwitz 2003, 292). Das hier beschriebene Verhältnis von Wissen bzw. generativer Wissensbasis (Kompetenz) und Handeln (Performanz) lässt sich auf einen prominenten, von Giddens eingeführten Begriff bringen: Dualität. Dieser Begriff vermeidet es, die Seiten einer Unterscheidung lediglich als Gegensätze zu verstehen und verschiedene Theorieperspektiven gegeneinander zu stellen. Vielmehr wird ihre wechselseitige Bedingtheit betont. Bei Giddens bezeichnet Dualität

«die allein analytisch unterschiedenen Momente der Wirklichkeit strukturierter sozialer Handlungssysteme. Strukturen selbst existieren gar nicht als eigenständige Phänomene räumlicher und zeitlicher Natur, sondern immer nur in der Form von Handlungen oder Praktiken menschlicher Individuen. Struktur wird immer nur wirklich in den konkreten Vollzügen der handlungspraktischen Strukturierung sozialer Systeme.» (Giddens 1988, 290)

Dies gilt auch für das, was oben als Kompetenz bezeichnet wurde. Nur im Handlungsvollzug ist Kompetenz unterstellbar (!) und der Vollzug selbst ist bereits in der Kompetenz angelegt. Insofern ist Kompetenz stets nur im Moment der Performanz; kommt also erst dann zur Wirklichkeit. Die möglichen Theorieanschlüsse zur Konzeption des Verhältnisses von Kompetenz und Performanz im Sinne der weiteren Ausarbeitung einer nominalen Definition lassen sich an dieser Stelle nur andeuten. Sie weiterzuverfolgen und getrennt von operationalen und normativen Ansprüchen zu denken, erscheint jedoch fruchtbar. Bezogen auf den Begriff der Medienkompetenz bedeutet das Gesagte: Erst im Medienhandeln selbst wird Medienkompetenz wirklich und indirekt als andere Seite von Performanz denkbar. Folgt man dieser Denkrichtung, ist es offensichtlich, warum sich dann letztlich auch Medienkompetenz nicht als Struktur zu erkennen gibt, sondern erst infolge von Wirklichkeitskonstruktionen zweiter Ordnung in operationalisierbaren Dimensionen messen und vermitteln lässt. Auf diesen Aspekt wird im folgenden Abschnitt eingegangen. 


\subsection{Wirklichkeitskonstruktionen erster und zweiter Ordnung}

Die in Abschnitt 3.1 vorgenommene nominale Bestimmung sollte die theoriegeleitete Definition von Kompetenz und Performanz aufgreifen und schärfen. Sie ist nun von den benannten operationalen und normativen Konzepten (vgl. Abschnitt 2.) abzugrenzen. Entsprechend des oben ausgearbeiteten nominalen Verständnisses von Kompetenz als mentale Realität der Handelnden ist Kompetenz nicht beobachtbar, sondern nur vermittelt durch die Rekonstruktion der Wirklichkeitskonstruktionen der in der Sozialwelt Handelnden zugänglich - so bereits bei Chomsky betont (vgl. Abschnitt 3.1; sowie auch Vonken 2017). Was beobachtet werden kann, ist Performanz. Und die bisherigen operationalen und normativen Kompetenzmodelle sind in diesem Sinne eigentlich Performanzmodelle (vgl. Vonken 2005). Aber auch die Beobachtung von Performanz bildet lebensweltliche Vollzüge nicht direkt ab, sondern nur vor dem Hintergrund jeweils eigener «Verfahrensregeln» des Beobachters (vgl. Schütz 1971, 7). Es handelt sich daher um eine Wirklichkeitskonstruktion zweiter Ordnung. Nicht nur der Wissenschaftler beobachtet in dieser Weise, sondern auch Pädagogen, Politiker etc., nach ihren jeweils eigenen Verfahrensregeln.

So beobachtet das Erziehungssystem nach der Eigenlogik vermittelbar/nichtvermittelbar (normativ) und besser/schlechter (operational). Es konstruiert dazu Handelnde als Kind bzw. Lebenslauf, d. h. als «semantische Einheit [...], die man von den Organismen und den psychischen Eigentümlichkeiten» der Erwachsenen unterscheiden muss (Luhmann 1991, 24; vgl. auch 2002, 82ff). Diese Eigenlogik des Systems spiegelt sich in den Kompetenzmodellen wieder, die darauf zielen, notwendige Kompetenzbereiche zu nennen und zu messen. So erklären sich dann auch unterschiedliche Kompetenz-Modelle vor dem Hintergrund der kulturgeschichtlich veränderbaren Wertesets des jeweiligen Bezugssystems (vgl. Vonken 2005, 133). Aus dieser Perspektive liegt es auf der Hand, dass sich auch Modelle der Medienkompetenz bzw. eigentlich Medienperformanz erstens an den jeweils vorherrschenden Werten der (Medien)Pädagogik und anderen gesellschaftlichen Teilsystem orientieren (vgl. Abschnitt 2), sowie zweitens an den konkreten Medienumgebungen der Gesellschaft (vgl. Abschnitt 4). Als Konstruktionen zweiter Ordnung beobachten sie nur bestimmtes Medienhandeln (und kein anderes) auf bestimmte Weise. 
Wissenschaft hat ebenfalls spezifische Verfahrensregeln, die als in besonderer Weise professionalisiert gelten können. Nichtsdestotrotz kann auch Wissenschaft nur rekonstruierte Konstruktionen liefern. Um dem Problem der Unmöglichkeit einer wertfreien Beobachtung zu entgehen, entwickelte Vonken «handlungstheoretisch begründete Kriterien zur Identifizierung kompetenten Handelns» (Vonken 2005, 133) und betrachtete dieses als «zu spezifizierenden Spezialfall» Habermas' kommunikativen Handelns (vgl. Vonken 2005, 170-178; insb. 173). Aber auch hier ist das Werteset eines wissenschaftlichen Bezugssystems Massstab für die Rekonstruktion von kompetentem Handeln und nicht die lebensweltliche Perspektive. Auch bei dem Versuch, die «Beurteilungskriterien auf eine allgemeiner gültige theoretische Grundlage zu stellen, kompetentes Handeln auf einer Metaebene der Beobachtung zu beurteilen» (Vonken 2005, 134) bleibt das Beobachterdilemma bestehen. Handlungstheoretisch (bzw. allgemein wissenschaftlich) begründete Beobachtungskriterien bleiben Konstruktionen zweiter Ordnung und unterscheiden sich in diesem Punkt nicht von denen anderer gesellschaftlicher Bezugssysteme (Erziehung, Politik, Wirtschaft). Konstruktionen zweiter Ordnung bilden Performanz also immer als Beobachterkonstrukt ab. Das Dilemma kann daher nicht gelöst, sondern nur bewusstgemacht und akzeptiert werden, indem die Differenz von eigenem und fremdem Bezugssystem betont wird ${ }^{2}$.

\section{Medienkompetenz als Kompetenz und Performanz in tiefgreifend mediatisierten Welten}

In den vorangegangenen Abschnitten wurden die Bedeutungsdimensionen von Kompetenz sortiert und der Kern einer Nominaldefinition herausgearbeitet. Kompetenz wurde sozialphänomenologisch bestimmt als Erzeugung von Situationen und Handeln aus dem Wissensvorrat. Es wurde dabei zudem auf die Dualität von Performanz und Kompetenz verwiesen,

2 Darin liegt auch eine Antwort auf die Frage Waldmanns und Aktas (2017, 102) nach der Konstitution der Medienpädagogik: Durch ein sozialphänomenologisches Verständnis von (Medien)kompetenz «kann die medienpädagogische Theoriebildung in einer Weise aufmerksam sein für ihre eigenen Ein- und Ausschlussprozeduren, die nicht zur Reproduktion von unternehmerischen Selbstverhältnissen ihrer Lern- und Bildungssubjekte führen». 
die also nicht getrennt, sondern in Ermöglichung zueinander zu denken sind. Gleichzeitig wurde die Perspektivität des Beobachtens von Performanz problematisiert.

Es bleibt zu fragen, was dies nun speziell für Medienkompetenz bedeutet. Bisher wurde Medienkompetenz in der Medienpädagogik grösstenteils als Teil einer allgemeinen Kompetenz mit dem speziellen Bezugsobjekt Medien gedacht. So wurde sie unter anderem beschrieben als formale Fähigkeiten (Befähigung zum Zeichengebrauch, technische Fähigkeiten, Kommunikations- und Sprachfähigkeiten, Wahrnehmungs-, Lese- und Rezeptionsfähigkeiten, Fähigkeiten zur Gestaltung von Medien) sowie Wissen über Medien (ihr Funktionieren, ihre Wirkungen, Produktions- und Verbreitungsformen) (vgl. Spanhel 2002, 5; vgl. für weitere Modelle zusammenfassend Süss et al. 2018, 114). In diesen Bestimmungen versammeln sich Zuschreibungen, die in Abschnitt zwei als operationale und normative Definitionen bestimmt wurden und schlussendlich die Perspektive des «Sollens» mitaufnehmen. Solche Definitionen wurden im Laufe des vorangegangenen Abschnitts abgelehnt. Kompetenz lässt sich aus der hier erarbeiteten theoretischen Perspektive nicht plausibel dimensionieren. Die Dimensionierung von Performanz nach eigenen Verfahrensregeln wurde als Beobachterartefakt bestimmt. Was aber nimmt der Medienkompetenzbegriff dann in den Blick?

De Medienthematik zeigt sich nicht als ein wohl umgrenzter Kompetenzbereich neben anderen, sondern als Aspekt von Kompetenz selbst. Statt also über Dimensionen von Kompetenzen und Medien nachzudenken, müsste eher gefragt werden: Was meint die Dualität von Kompetenz in einer durch digitale Technik geprägten Welt? (vgl. auch Heinen und Kerres 2017, 130) Diese Frage verknüpft medienpädagogische und kommunikationswissenschaftliche Forschung auf andere Weise als die nach einer medienbezogenen Kompetenz. Sie fragt nach den Bedingungen einer Erzeugung von Situation und Handeln (Kompetenz) in einer digitalisierten bzw. «tiefgreifend mediatisierten Welt» und nicht nach Medienwissen als Voraussetzung für Medienhandeln. Hierzu lassen sich bereits zwei Forschungsansätze identifizieren, die es stärker zu verknüpfen gilt: ein kommunikationswissenschaftlicher und ein bildungswissenschaftlicher. Tiefgreifend mediatisierte Welten sind Gegenstand des von Andreas Hepp 
und Friedrich Krotz begründeten Mediatisierungsansatzes. Er beschreibt die zunehmende Prägung gesellschaftlicher Praktiken und Sinndeutungsprozesse durch Medien. Es wird davon ausgegangen, dass sich Mediatisierung in Wellen (Couldry und Hepp 2017) vollzieht. Diese Wellen sind dabei nicht, wie in anderen Ansätzen, als revolutionärer Wechsel der Dominanz eines spezifischen Mediums gekennzeichnet, sondern durch entscheidende qualitative Veränderung der Medienumgebung, verstanden als «the totaly of communications media available at one point in space-time» (ebd, 39). Der gegenwärtige (dritte) Mediatisierungsschub wird dabei als Digitalisierung bezeichnet (vgl. Couldry und Hepp 2017). Er unterscheidet sich von früheren Phasen durch eine Medienumgebung, die durch eine starke technische Verwobenheit kennzeichnet ist. (vgl. Couldry und Hepp 2017, 53-55). Zugleich gehen die Autoren mit dem Digitalzeitalter von einer neuen Dimension der Mediatisierung innerhalb der letzten 150 Jahre aus, die sie mit der Erweiterung «deep mediatization» (ebd., 37) deutlich machen. Die Kennzeichen einer tiefgreifenden Mediatisierung arbeiten sie heraus, indem sie die Frage nach der Konstruktion der sozialen Realität, fünfzig Jahre nach Berger und Luckmann (1967), neu beantworten: «the concept of 〈deep〉 mediatization [...] involves a fundamental transformation in how the social world is constructed» (Couldry und Hepp 2017, 7). Bereits der Titel der Publikation verrät, wie diese Transformation gedacht wird. Es handelt sich um eine mediatisierte Konstruktion.

«We do mean by this, that the social world has significantly more complexity, when its forms and patterns are, in part, sustained in and through media and their infrastructures. [...] the horizon of our practices is a social world for which media are fundamental reference-points and resources.» (Couldry und Hepp 2017, 15)

Medien definieren sie dabei als «technologically based media of communication which institutionalize communication» (ebd., 32). Primäre $\mathrm{Me}-$ dien wie die Sprache, die bei Berger und Luckmann eine Rolle spielte, aber auch symbolisch generalisierte Kommunikationsmedien (Geld, Macht, Liebe) schliessen sie aus ihrer Definition aus. Statt primäre und sekundäre Medien aber starr voneinander abzugrenzen, gilt es vielmehr Medialität als Bedingung menschlichen Handelns zu begreifen. Dieses Argument nimmt 
ein zweiter Ansatz in den Blick, und zwar der der strukturalen Medienbildung. So betonen Fromme, Iske und Marotzki (2011, 7), dass «Sozialisation immer medial vermittelt oder mindestens medial induziert stattfindet», «Medialität [also] in alle Sozialisationsprozesse prinzipiell eingeschrieben ist». Die Ausarbeitung eines Medialitätsverständnisses sowie eines entsprechenden Analysefokus bezeichnen sie als «Königsweg zur Analyse von Sozialisations-, Lern- und Bildungsprozessen» (Fromme, Iske, und Marotzki 2011, 7). Oder: Bleibt man bei dem Kompetenzbegriff, wofür der Beitrag einige Argumente liefert, als Königsweg zur Analyse der Dualität von Kompetenz in tiefgreifend mediatisierten Welten. Es gilt, sich nicht an realhistorischen Bedingungen einzelner Medien zu orientieren und damit einzelnen technischen Innovationen hinterherzulaufen, sondern Medialität als Aspekt von Kompetenz zu reflektieren. Ähnlich betont es Meder für Bildungsprozesse: Für ihn ist das «Medium - die Sprache oder das Internet - [...] die Art und Weise, wie Bildung faktisch wird» (Meder 2014, 69). Es ist das Substrat, in dem sich Bildung vollzieht, so dass «Bildung stets Medienbildung ist» (Meder 2014, 65) oder eben Kompetenz immer schon Medienkompetenz. Diese Ideen können Kommunikations- und Bildungswissenschaft gemeinsam als Kern eines theoretisch begründeten Kompetenzbegriffs in tiefgreifend mediatisierten Welten weiter ausarbeiten.

\section{5. $\quad$ Fazit}

Gerade vor dem Hintergrund aktueller Diskussionen zur Digitalisierung kann eine Revitalisierung des Medienkompetenzkonzepts unter der Voraussetzung begrifflicher Klarheit für die Medienpädagogik lohnenswert sein. Dazu müssen die einzelnen Denkweisen jedoch auseinandergehalten werden.

Eine wissenschaftlich theoriegeleitete Definition muss sich künftig mit Fragen zur Medialität von Wirklichkeitskonstruktionen durch Handelnde und den diesen Konstruktionen zugrundeliegenden Bedingungen tiefgreifend mediatisierter Welten auseinandersetzen, statt sich mit der Reifizierung von Kompetenz- bzw. Performanzmodellen zu befassen. Eine solche Definition kommt in dem hier vorgestellten Sinn als Dualität von Kompetenz ohne systemisch bedingte Klassifikationen und Imperative aus. 
Hierdurch kann der Begriff selbst als auch «die Betroffenen von Kompetenzentwicklung» von normativen Ansprüchen entlastet werden, da «auf diese Weise kein 〈Zugriff〉 auf Eigenschaften ihrer Persönlichkeit erfolgen muss» (Vonken 2005, 191). Das hat allerdings zur Folge, «dass sich die Entwicklung von Kompetenz in Lehr-Lernprozessen nicht sicherstellen, sich Kompetenz nicht trainieren lässt» (ebd., 187) und damit die Legitimation medienpädagogischer Bemühungen in Frage gestellt werden könnte (vgl. auch Moser 2003, 27). Es ist mitnichten das Ansinnen des Beitrags eine solche Deligitimation zu befördern, wohl aber soll dafür plädiert werden, normative Ansprüche von theoriegeleiteten Diskussionen zu unterscheiden. Begriffe müssen dazu geschärft und Beobachtungslogiken klar gemacht werden.

So schliessen von der Medienpädagogik aus der Logik des Erziehungssystems heraus normativ begründete Medien`kompetenzen〉 eigentlich an die Performanzseite des Theoriebegriffs an, ohne dass dem bisher hinreichend Rechnung getragen wurde. Eine stärker an der Performanzseite der Dualität von Kompetenz orientierte Medienpädagogik kann ihre normativen Ansprüche aufrecht erhalten, ohne sich die Kritik einzuhandeln, dass sich Kompetenzen nicht vermitteln bzw. intentional entwickeln lassen, sondern als erfahrungsbasierte Konstruktionsleistungen der Handelnden (vgl. Vonken 2005, 52, 187) zu verstehen sind. Diese Konstruktionsleistungen sind im Sinne einer sozialphänomenologischen Perspektive als Prozess des fortlaufenden Wissenserwerbs zu denken. Dieser erfolgt über Erfahrungen von Routine- und problematischen Situationen und ist an die Bestimmung aktueller Situationen in der Performanz gebunden. Während durch Erfahrungen, die «fraglos〉 ablaufen, der Wissensvorrat bestätigt wird, aber 〈nichts Neues〉 entsteht ermöglichen als problematisch erfahrene Situationen die «Sedimentierung 〈neuer〉 Auslegungen» (Schütz und Luckmann 2003, 178f.). Insofern kann es Aufgabe der performanzorientierten Medienpädagogik sein, Erfahrungsräume für sowohl unproblematische als auch «problematische» Erfahrungen, d. h. allgemein Performanzmöglichkeiten zu kreieren und damit «zum einen die Kenntnis des generischen Regelwerkes sicher zu stellen und zum anderen dabei zu helfen, die psychischen Störfaktoren unter Kontrolle zu halten, damit optimale - wenn schon nicht ideale - Performanz stattfinden kann» (Meder 2011, 76). 
Auch operationale Denkweisen knüpfen eigentlich an die Performanzseite an und auch sie müssen die Logik ihrer Konstruktionen klar machen. Handelt es sich um normativ begründete Messmodelle oder Annäherungen an die theoretisch begründete Dualität von Kompetenz. Während sich erstere an Bildungsstandards und damit an normativen Erwartungen des Erziehungssystems orientieren, müssten zweitere an die Konstruktionen erster Ordnung der in der Sozialwelt Handelnden anknüpfen. Sie sind zwar keiner direkten Beobachtung zugänglich. Aber durch stärker qualitative sozialwissenschaftliche Verfahren lässt sich auch Performanz als Binnenperspektive der Handelnden verstehend rekonstruieren. Eine solche Form der empirischen Forschung müsste gestärkt werden. Normativ begründete Messmodelle liefern die bekannten Vergleichsstudien und sind Grundlage für bildungspolitische Bemühungen. Verstehende Rekonstruktion hingegen führt näher an die Dualität von Kompetenz. Von daher kann ein Verständnis von Medienkompetenz als Dualität von Kompetenz und Performanz für den «NNew Look〉 der Medienpädagogik» (Heinen und Kerres 2017, 130) im Sinne einer tragfähigen Unterscheidung unterstützend wirken.

\section{Literatur}

Aebli, Hans. 1980. Denken: Das Ordnen des Tuns. Stuttgart: Klett-Cotta.

Arnold, Karl-Heinz, und Carola Lindner-Müller. 2011. «Stichwort Kompetenz». In Klinkhardt Lexikon Erziehungswissenschaft (KLE), herausgegeben von KlausPeter Horn, Heidemarie Kemnitz, Winfried Marotzki und Uwe Sandfuchs, 230-31 Bd. 2: Bad Heilbrunn: Klinkhardt.

Baacke, Dieter. 1980. Kommunikation und Kompetenz: Grundlegung einer Didaktik der Kommunikation und ihrer Medien. 3. Aufl. München: Juventa-Verlag.

Baacke, Dieter. 1996. «Medienkompetenz - Begrifflichkeit und Sozialer Wandel». In Medienkompetenz als Schlüsselbegriff, herausgegeben von Antje von Rein, 112-24. Bad Heilbrunn: Klinkhardt.

Chomsky, Noam. 1970. Aspekte der Syntax-Theorie. With the assistance of E. Lang. Sammlung Akademieverlag: Sprache 11. Berlin: Akademie-Verl.

Couldry, Nick, und Andreas Hepp. 2017. The Mediated Construction of Reality. 1. Auflage. Cambridge: Polity Press. 
Fromme, Johannes, Iske, Stefan, und Winfried Marotzki. 2011. «Zur konstitutiven Kraft der Medien - Einleitung». In Medialität und Realität. Zur konstitutiven Kraft der Medien, herausgegeben von Johannes Fromme, Stefan Iske, und Winfried Marotzki, 7-12. Wiesbaden: VS Verlag. https://doi.org/10.1007/978-3-53192896-8_1.

Gapski, Harald. 2001. Medienkompetenz: Eine Bestandsaufnahme und Vorüberlegungen zu einem systemtheoretischen Rahmenkonzept. 1. Aufl. Wiesbaden: Westdt.Verlag.

Giddens, Athony. 1988. «Die ,Theorie der Strukturierung': Ein Interview mit Anthony Giddens». Zeitschrift für Soziologie 17 (4): 286-95.

Grundmann, Matthias. 2017. «Sozialisation - Erziehung - Bildung: Eine kritische Begriffsbestimmung». In Lehrbuch der Bildungssoziologie, herausgegeben von Rolf Becker. 3., aktualisierte und überarbeitete Auflage, 63-88. Wiesbaden: Springer VS. https://doi.org/10.1007/978-3-658-15272-7_3.

Heinen, Richard, und Michael Kerres. 2017. «Bildung in der digitalen Welt» als Herausforderung für Schule». DDS - Zeitschrift für Erziehungswissenschaft, Bildungspolitik und pädagogische Praxis 109 (2): 128-45. https://learninglab.unidue.de/sites/default/files/heinen-kerres-dds.pdf.

Hugger, Kai-Uwe. 2008. «Medienkompetenz». In Handbuch Medienpädagogik, herausgegeben von Uwe Sander, Frederike von Gross, und Kai-Uwe Hugger, 93-99. Wiesbaden: VS Verlag für Sozialwissenschaften. https://doi.org/10.1007/978-3531-91158-8_10.

Jarren, Otfried, und Christian Wassmer. 2009. «Medienkompetenz. Begriffsanalyse und Modell. Ein Diskussionsbeitrag zum Stand der Medienkompetenzforschung». merz | medien + erziehung 53 (3): 46-51. https://doi.org/10.5167/uzh20055.

Kerres, Michael. 2017. «Digitalisierung als Herausforderung für die Medienpädagogik. 〈Bildung in einer digital geprägten Welt〉. In Pädgogischer Mehrwert? Digitale Medien in Schule und Unterricht, herausgegeben von Christian Fischer. 85-104. Münster: Waxmann Verlag.

Knaus, Thomas. 2018. «[Me]nsch - Werkzeug - [I]nteraktion. Theoretisch-konzeptionelle Analysen zur 〈Digitalen Bildung) und zur Bedeutung der Medienpädagogik in der nächsten Gesellschaft». Herausgegeben von Jasmin Bastian, Tobias Feldhoff, Marius Harring, und Klaus Rummler. MedienPädagogik: Zeitschrift für Theorie und Praxis der Medienbildung 31 (〈Digitale Bildung〉. Medienbezogene Bildungskonzepte für die (nächste Gesellschaft)): 1-35. https://doi. org/10.21240/mpaed/31/2018.03.26.X.

Meder, Norbert. 2011. «Von der Theorie der Medienpädagogik zu einer Theorie der Medienbildung». In Medialität und Realität. Zur konstitutiven Kraft der Medien, herausgegeben von Johannes Fromme, Stefan Iske, und Winfried Marotzki, 67-81. Wiesbaden: VS Verlag für Sozialwissenschaften. https://doi. org/10.1007/978-3-531-92896-8_5. 
Meder, Norbert. 2014. «Das Medium als Faktizität der Wechselwirkung von Ich und Welt (Humboldt)». In Perspektiven der Medienbildung, herausgegeben von Winfried Marotzki und Norbert Meder, 45-69. Wiesbaden: Springer Fachmedien Wiesbaden. https://doi.org/10.1007/978-3-658-03529-7_3.

Moser, Heinz. 2003. «Von der Medienkompetenz zur Medienbildung. Diskurstheoretische Überlegungen». Medienwissenschaft Schweiz 2/2003: 26-34.

Schütz, Alfred, und Thomas Luckmann. 2003. Strukturen der Lebenswelt. UTB 2412. Konstanz: UVK-Verl.-Ges.

Schütz, Alfred. 1971. Gesammelte Aufsätze. Bd. 1. Das Problem der sozialen Wirklichkeit. Den Haag: Martinus Nijhoff.

Spanhel, Dieter. 2002. «Medienkompetenz als Schlüsselbegriff der Medienpädagogik?» forum medienethik 1 (Medienkompetenz-Kritik einer populären Universalkonzeption): 48-53. https://www.lmz-bw.de/fileadmin/user_upload/ Downloads/Handouts/spanhel-medienkompetenz-schluesselbegriff-medienpaedagogik.pdf.

Spanhel, Dieter. 2011. «Medienbildung als Grundbegriff der Medienpädagogik. Begriffliche Grundlagen für eine Theorie der Medienpädagogik». In Medienbildung und Medienkompetenz. Beiträge zu Schlüsselbegriffen der Medienpädagogik, herausgegeben von Heinz Moser, Petra Grell, und Horst Niesyto, 95-120. München: kopaed. https://doi.org/10.21240/mpaed/20/2011.09.15.X.

Süss, Daniel, Claudia Lampert, und Christine W. Trültzsch-Wijnen, Hrsg. 2018. Medienpädagogik: Ein Studienbuch zur Einführung. 3. Auflage. Lehrbuch. Wiesbaden: Springer VS. https://doi.org/10.1007/978-3-658-19824-4.

Sutter, Tilmann. 2014. "Selbstsozialisation und Medienbildung». In Perspektiven der Medienbildung, herausgegeben von Winfried Marotzki und Norbert Meder, 71-90. Medienbildung und Gesellschaft Bd. 27. Wiesbaden: Springer VS. https://doi.org/10.1007/978-3-658-03529-7_4.

Theunert, Helga. 2011. «Aktuelle Herausforderungen für die Medienpädagogik». Aus Politik und Zeitgeschichte, Nr. 3: 24-29. http://www.bpb.de/publikationen/ QBHoGU,o,Aktuelle_Herausforderungen_f\%FCr_die_Medienp\%E4dagogik. html.

Theunert, Helga, und Bernd Schorb. 2010. «Sozialisation, Medienaneignung und Medienkompetenz in der mediatisierten Gesellschaft». In Die Mediatisierung der Alltagswelt, herausgegeben von Maren Hartmann und Andreas Hepp, 243254. Wiesbaden: Springer VS. https://doi.org/10.1007/978-3-531-92014-6_16.

Tulodziecki, Gerhard. 2011. «Zur Entstehung und Entwicklung zentraler Begriffe bei der pädagogischen Auseinandersetzung mit Medien». Herausgegeben von Heinz Moser, Petra Grell, und Horst Niesyto. MedienPädagogik: Zeitschrift für Theorie und Praxis der Medienbildung 20 (Medienbildung im Spannungsfeld medienpädagogischer Leitbegriffe): 11-39. München: kopaed. https://doi. org/10.21240/mpaed/20/2011.09.11.X.

Vonken, Matthias. 2005. Handlung und Kompetenz. Zugl. Erfurt, Univ., Diss., 2004, Wiesbaden: VS Verl. für Sozialwiss. 
Waldmann, Maximilian, und Ulas Aktas. 2017. «Lernen und Bildung als Erfahrungsgeschehen in Vollzügen des digital Medialen. Phänomenologische und diskurstheoretische Nachfragen zu Grundbegriffen und Konstitution der Medienpädagogik». Herausgegeben von Christian Swertz, Wolfgang B. Ruge, Alexander Schmölz, und Alessandro Barberi. MedienPädagogik: Zeitschrift für Theorie und Praxis der Medienbildung 29 (Konstitution der Medienpädagogik): 89-104. https://doi.org/10.21240/mpaed/29/2017.09.04.X.

Weiner, Joachim. 2011. «Medienkompetenz〉 - Chimäre oder Universalkompetenz?. Essay». Aus Politik und Zeitgeschichte, Nr. 3: 42-46. http://www.bpb. de/publikationen/F7DNBQ,O,Medienkompetenz_Chim\%E4re_oder_Universalkompetenz_Essay.html.

Wirth, Uwe. 2002. «Der Performanzbegriff im Spannungsfeld von Illokution, Iteration und Indexikalität». In Performanz. Zwischen Sprachphilosophie und Kulturwissenschaften, herausgegeben von Uwe Wirth, 9-62. Frankfurt a. M.: Suhrkamp. 